\title{
Identification and Characterization of Verticillium nonalfalfae-Responsive MicroRNAs in the Roots of Resistant and Susceptible Hop Cultivars
}

\author{
Urban Kunej ${ }^{1}$, Jernej Jakše ${ }^{1} \mathbb{1}$, Sebastjan Radišek ${ }^{2}$ (I) and Nataša Štajner ${ }^{1, * \mathbb{C}}$ \\ 1 Department of Agronomy, Biotechnical Faculty, University of Ljubljana, 1000 Ljubljana, Slovenia; \\ urban.kunej@bf.uni-lj.si (U.K.); jernej.jakse@bf.uni-lj.si (J.J.) \\ 2 Plant Protection Department, Slovenian Institute of Hop Research and Brewing, 3310 Žalec, Slovenia; \\ sebastjan.radisek@ihps.si \\ * Correspondence: natasa.stajner@bf.uni-lj.si; Tel.: +38-613-203-255
}

check for

updates

Citation: Kunej, U.; Jakše, J.;

Radišek, S.; Štajner, N. Identification and Characterization of Verticillium

nonalfalfae-Responsive MicroRNAs in the Roots of Resistant and Susceptible Hop Cultivars. Plants 2021, 10, 1883. https://doi.org/10.3390/plants10091883

Academic Editors: Vesselin Baev,

Mariyana Gozmanova and

Jean Molinier

Received: 19 July 2021

Accepted: 9 September 2021

Published: 11 September 2021

Publisher's Note: MDPI stays neutral with regard to jurisdictional claims in published maps and institutional affiliations.

Copyright: (c) 2021 by the authors. Licensee MDPI, Basel, Switzerland. This article is an open access article distributed under the terms and conditions of the Creative Commons Attribution (CC BY) license (https:/ / creativecommons.org/licenses/by/ $4.0 /)$.

\begin{abstract}
MicroRNAs are 21- to 24-nucleotide-long, non-coding RNA molecules that regulate gene expression at the post-transcriptional level. They can modulate various biological processes, including plant response and resistance to fungal pathogens. Hops are grown for use in the brewing industry and, recently, also for the pharmaceutical industry. Severe Verticillium wilt caused by the phytopathogenic fungus Verticillium nonalfalfae, is the main factor in yield loss in many crops, including hops (Humulus lupulus L.). In our study, we identified 56 known and 43 novel miRNAs and their expression patterns in the roots of susceptible and resistant hop cultivars after inoculation with $V$. nonalfalfae. In response to inoculation with $V$. nonalfalfae, we found five known and two novel miRNAs that are differentially expressed in the susceptible cultivar and six known miRNAs in the resistant cultivar. Differentially expressed miRNAs target 49 transcripts involved in protein localization and pigment synthesis in the susceptible cultivar, whereas they are involved in transcription factor regulation and hormone signalling in the resistant cultivar. The results of our study suggest that the susceptible and resistant hop cultivars respond differently to $V$. nonalfalfae inoculation at the miRNA level and that miRNAs may contribute to the successful defence of the resistant cultivar.
\end{abstract}

Keywords: Humulus lupulus; Verticillium nonalfalfae; biotic stress; microRNA; high-throughput sequencing

\section{Introduction}

Hops (Humulus lupulus L.) are traditionally cultivated for use in the brewing industry as an essential ingredient that provides flavour and acts as a stabilizer or preserver of the beer [1]. In recent years, the bioactive compounds of hops have also become increasingly attractive for use in the pharmaceutical industry [2,3]. One of the main limiting factors in hop production are fungal diseases, especially those caused by the soil-borne plant pathogenic fungus Verticillium nonalfalfae (formerly known as Verticillium albo-atrum) [4]. The symptoms of Verticillium wilt in hops caused by $V$. nonalfalfae vary depending on the pathogenicity of the fungal strain and the sensitivity of the cultivar. Susceptible hop cultivars can suffer from severe symptoms (e.g., leaf chlorosis and necrosis) and also complete dieback of rootstock caused by a highly virulent strain of $V$. nonalfalfae [5].

To prevent infections with various phytopathogens, plants have evolved multi-tiered defence mechanisms. The first layer of defence is represented by pattern recognition receptors (PRRs) present at the cell membrane surface that recognize conserved pathogenassociated molecular patterns (PAMPs). This phenomenon is called PAMP-triggered immunity (PTI) or basal defence. PTI comprises both physical and chemical defence responses, e.g., the deposition of lignin-like compounds in the cell wall, the production of reactive oxygen species (ROS) and the activation of signalling cascades that modulate 
gene expression. Successful pathogens can counteract the plant basal immune response by deploying the effectors into the cytoplasm of plant cells to attenuate defence. Their presence in the cytoplasm is directly or indirectly detected by receptors, so-called resistance (R) proteins, or nucleotide-binding leucine-rich repeat (NB-LRR) proteins encoded by $R$-genes. Resistance mediated by $R$-genes is considered to be the second layer of defence and confers an enhanced type of defence, known as effector-triggered immunity (ETI) [6,7]. To achieve the best effectiveness of the defence response, all defence mechanisms must be well regulated. In this dynamic and complex network of gene regulatory pathways during the immune response, short non-coding RNAs, so-called microRNAs (miRNAs), play a pivotal role [8,9]. miRNAs are a class of small endogenous non-coding RNA molecules with a length of 21 to 24 nucleotides, which act as post-transcriptional regulators of gene expression and are thus involved in various biological processes [10]. Recently, Soto-Suarez et al. [11] demonstrated that miR396 mediates the PAMP-triggered immune response against necrotrophic and hemibiotrophic fungal pathogens in Arabidopsis. Navarro et al. [12] discovered that in Arabidopsis plants treated with PAMP (Flagellin fragment 22), miR393 was upregulated and, as a result, the expression of F-box auxin receptors was silenced, leading to suppression of the auxin signalling pathway and enhanced PTI. The upregulation of miR393 was also detected in soybean in response to infection with the pathogenic fungus Phytophthora sojae. Moreover, miR393-knockdown soybean plants show increased susceptibility to infection [13]. However, miRNAs other than miR393 can modulate the auxin signalling pathway in various pathogen infections and are involved in plant immune response. For example, miR160 regulates auxin response factors (ARFs) in potato and, thereby, indirectly affects the expression of genes that modulate salicylic acidauxin cross-talk, which is associated with local defence and systemic acquired resistance to Phytophthora infestans [14]. NB-LRR proteins, products of $R$-genes that mediate ETI in plants, are targeted by several miRNAs, such as miR2118 in Medicago truncatula [15] and in cotton infected with Verticillium dahliae [16], by gma-miR1510a/b, which contributes to the resistance to Phytophthora sojae [17], and by ptc-MIR482, ptc-MIR1447 and ptc-MIR1448 in Populus trichocarpa [18,19].

Although there is increasing evidence that miRNAs play an important role in the regulation of gene expression during the immune response in plants, there is a scarcity of information on miRNA-mediated gene silencing during the pathogenesis of fungal diseases, such as Verticillium wilt, in various crops. In the resistant hop cultivar Wye Target, a single quantitative trait locus (QTL) has been identified, which explains 26\% of the phenotypic variance for Verticillium wilt resistance [20], and potential resistance gene analogue-expressed sequence tag (RGA-EST) markers have been developed [21]. A well-studied example of Verticillium wilt resistance to date relates to the tomato's Ve1 gene, which codes for a leucine-rich repeat (LRR) receptor-like protein that confers the resistance to a strain of $V$. dahlia race 1 or $V$. nonalfalfae [22-25]. Ve1 orthologue was also characterized in hops and it is suggested that it provides the resistance to $V$. dahliae strain 1 by detecting an effector protein Ave1 [26]. In a proteomic study, Mandelc et al. [27] observed an accumulation of defence-related proteins, such as chitinase, $\beta$-glucanase, thaumatin-like protein, peroxidase and germin-like proteins in the compatible interactions between $V$. nonalfalfae and hops, while such a response was not detected in the incompatible interactions. Similarly, an increased expression of genes is involved in innate immunity; the jasmonic acid pathway and wounding was observed in the roots and shoots of the susceptible hop cultivar [28], while Cregeen et al. [29] observed the increased expression of genes involved in ubiquitination (SKP1), vesicle trafficking (cdc48), protein degradation (puromycin-sensitive aminopeptidase), protein-protein interactions (syntaxin and Fk506), transport (acyl-CoA-binding protein) and morphogenesis (furry protein) in the resistant cultivar and decreased expression in the susceptible cultivar.

In the present work, we characterized miRNAs in hops and identified $V$. nonalfalfaeresponsive miRNAs in the roots of the susceptible and resistant hop cultivars. Furthermore, we used an in silico approach to predict transcripts targeted by $V$. nonalfalfae-responsive 
miRNAs and discussed their potential role in the defence response to $V$. nonalfalfae in susceptible or resistant hop cultivars based on their interactions within a local proteinprotein interaction network cluster and their gene ontology.

\section{Results}

\subsection{High-Throughput Sequencing of H. lupulus miRNAs}

To investigate the miRNA response in roots of susceptible and resistant hop cultivars after inoculation with $V$. nonalfalfae, small RNA libraries were constructed from three control and three inoculated root samples of both hop cultivars. A total of 90,355,033 reads were obtained, ranging from 5,222,013 to 10,059,037 reads per library with a mean read length of $14 \mathrm{bp}$ to $20 \mathrm{bp}$. After processing the raw sequencing data, we obtained from 1,771,295 to $4,636,681$ sequencing reads with a mean read length of $18 \mathrm{bp}$ to $22 \mathrm{bp}$ per library. Processed reads from each library were aligned against the hop draft genome [30,31] disallowing mismatches (Table 1).

Table 1. Statistics of small RNA sequencing reads from 12 sRNA libraries. First two letters of sample names denote cultivar; CE, susceptible cultivar Celeia; WT, resistant cultivar Wye Target; Ctrl, control; Inoc, inoculated, and numbers denote biological replicates (1-3).

\begin{tabular}{cccccc}
\hline Sample & $\begin{array}{c}\text { Num. of Raw } \\
\text { Reads }\end{array}$ & $\begin{array}{c}\text { Mean Length of } \\
\text { Raw Reads (bp) }\end{array}$ & $\begin{array}{c}\text { Num. of Reads } \\
\text { after Processing }\end{array}$ & $\begin{array}{c}\text { Mean Length of } \\
\text { Processed Reads (bp) }\end{array}$ & $\begin{array}{c}\text { Reads Mapped to } \\
\text { Hop Genome }\end{array}$ \\
\hline CE-Ctr11 & $10,041,349$ & 18 & $4,636,681$ & 20 & $2,634,528(56.82 \%)$ \\
\hline CE-Ctr12 & $6,109,522$ & 16 & $2,232,764$ & 20 & $1,341,656(60.09 \%)$ \\
\hline CE-Ctrl3 & $8,146,318$ & 17 & $3,232,808$ & 20 & 19 \\
\hline CE-Inoc1 & $8,631,607$ & 16 & $2,672,954$ & 20 & $1,575,284(58.93 \%)$ \\
\hline CE- Inoc2 & $6,991,916$ & 18 & $3,466,372$ & $2,000,154(57.70 \%)$ \\
\hline CE- Inoc3 & $6,723,592$ & 14 & $1,771,295$ & 22 & $1,230,008(69.44 \%)$ \\
\hline WT-Ctrl1 & $6,223,982$ & 20 & $3,745,411$ & 21 & $2,094,410(55.92 \%)$ \\
\hline WT-Ctrl2 & $5,222,013$ & 19 & $2,933,213$ & 22 & $1,729,720(58.97 \%)$ \\
\hline WT-Ctrl3 & $7,737,001$ & 20 & $4,083,377$ & $2,393,417(58.61 \%)$ \\
\hline WT-Inoc1 & $10,059,037$ & 18 & $4,435,862$ & 21 & $2,559,244(57.69 \%)$ \\
\hline WT-Inoc2 & $7,537,943$ & 18 & $3,698,547$ & 20 & $2,125,103(57.46 \%)$ \\
\hline WT-Inoc3 & $6,930,753$ & 20 & $3,202,208$ & 22 & $1,811,863(56.58 \%)$ \\
\hline
\end{tabular}

\subsection{Known and Novel miRNAs Identified in Hop Root Tissue}

Reads perfectly aligned against the hop genome were subjected to miRNA prediction analysis using the miR-PREFeR pipeline [32], which predicted 2591 MIR loci (miRNA candidates) and their mature, precursor and star miRNA sequences. Of the 2591 miRNA candidates, 120 aligned with known miRNAs from miRBase [33] and 2471 miRNA candidates were novel. Of the 120 known miRNA candidates identified, 100 miRNA candidates encoded 44 different mature miRNAs (miR) that perfectly aligned with known miRNAs that belong to 27 different miRNA families (MIR) from miRBase [33], and 20 miRNA candidates encoded 12 different mature miRNAs that aligned with up to two mismatches against known miRNAs belonging to 10 different miRNA families (Figure 1, Table S1). Aligning-predicted miRNA precursor sequences (pre-miRNAs) against RNA sequences deposited in the RNAcentral database [34] did not result in additional annotations. The names of known miRNAs identified in H. lupulus were assigned based on the criteria and conventions for miRNA naming (Supplementary Table S1) [33,35]. Identified known miRNA families were not evenly represented in the number of members. The most represented families were MIR169 with five members; MIR156 and MIR477 with four members; MIR160, MIR167, MIR171 and MIR319 with three members; MIR159, MIR172, MIR390, 
MIR393, MIR394, MIR395, MIR399 and MIR482 with two members and the remaining 15 families were represented by a single member (Table S1). Additionally, the same mature miRNAs of the same family derive from a different number of precursor miRNAs or MIR loci. For example, MIR169 members derive from two (hlu-miR169m, hlu-miR169n) to six (hlu-miR169g, hlu-miR169h, hlu-miR169i, hlu-miR169j, hlu-miR169k, hlu-miR169l) different miRNA precursors and are altogether coded by 16 MIR loci.

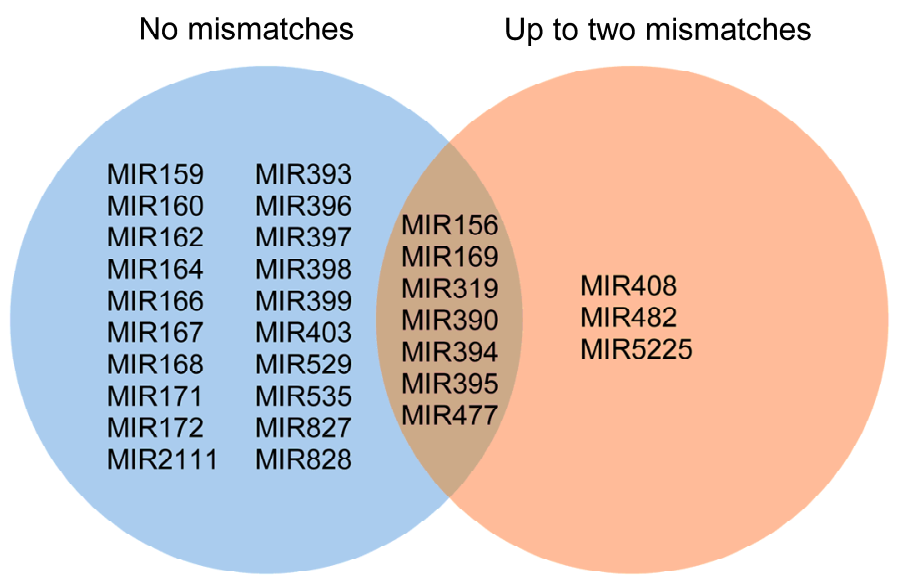

Figure 1. Families of miRNAs to which belong predicted hop miRNAs that align with known miRNAs deposited in miRBase. Hop miRNA families with members that align without mismatches are in the blue circle (20 miRNA families) and those that align with up to two mismatches are in the orange circle ( 3 miRNA families). Seven families comprise members that align perfectly or with up to two mismatches.

Seven miRNA families comprise miRNAs that align perfectly or with up to two mismatches with known miRNAs of the corresponding miRNA family deposited in miRBase. Hlu-miR169o-p, hlu-miR319g-i, hlu-miR390b-c, hlu-miR394c-d, hlu-miR395c, hlumiR477e, hlu-miR482a-b and hlu-miR482c aligned with one mismatch against known miRNAs from miRBase and are also very similar to other members of their family. With two mismatches, the following miRNAs were aligned against known miRNAs from miRBase; hlu-miR156g, hlu-miR477c-d, hlu-miR5225 and hlu-miR408a-b (Figure 1).

The most abundant miRNAs in the susceptible cultivar were hlu-miR482a-b and hlu-miR482c with, on average, 26,633 and 33,356 normalized read counts, respectively, followed by hlu-miR159a-b with, on average, 23,689 normalized read counts. Aforementioned miRNA families were also the most abundant in the resistant cultivar; hlu-miR482a-b had on average 30,089, hlu-miR482c had 29,664 and hlu-miR159a-b had 17,212 normalized read counts.

Of the 2471 predicted novel miRNA candidates, those with at least 100 reads mapped to the predicted mature miRNA and at least one read to the corresponding star miRNA, were considered as highly reliable predictions. Thus, we obtained 43 reliable predictions of novel mature miRNAs derived from 152 miRNA precursors. The names of novel mature miRNAs identified in H. lupulus were given as "miRNA-" followed by a consecutive number of the prediction but are not in order because unreliable predictions have been removed (Supplementary Table S2). Where novel predicted mature miRNAs were derived from different precursor sequences, these miRNAs were assigned two or more slashseparated precursor names, e.g., miRNA-363/miRNA-1427.

Using CD-HIT-EST [36] to cluster precursor sequences of novel miRNAs and premiRNAs from miRBase, we identified $89.01 \%$ similarity between the predicted precursor of miRNA-405 and miR156v (MI0022992) from Malus domestica. Furthermore, the mature sequence of miRNA-405 aligned with three mismatches (e-value: 0.36) against csi-miR156g$3 p$ (MIMAT0048860) from miRBase. Based on this evidence, we assigned the novel miRNA405 to the MIR156 family and named it as hlu-miR156h. 
Additionally, clustering precursor sequences showed $81.37 \%$ similarity between one novel miRNA derived from four different precursors (miRNA-665, miRNA-2226, miRNA2474, miRNA-2537) and miR395j from P. trichocarpa (MI0002324). These miRNA precursors were assigned to the MIR395 family, whereas the mature miRNA was treated as novel. The remaining novel miRNA precursors were clustered into thirty-three clusters, which were treated as novel miRNA families (Table S2).

\subsection{Differentially Expressed miRNAs between $V$. nonalfalfae-Inoculated and Control Samples}

The differential expression analysis of known and novel predicted mature miRNAs between inoculated and control samples was performed separately for each cultivar. miRNAs having at least 100 read counts in the susceptible or resistant cultivar were included in the differential expression analysis. miRNAs with the FDR corrected $p$-value $\leq 0.1$ were considered as significantly differentially expressed. The results of differential expression analysis indicate that different members of the same miRNA family differ significantly in expression levels (Supplementary Table S1). Furthermore, except for hlu-miR477f and hlu-miR159c-d, which show similar expression patterns in the susceptible and resistant cultivars inoculated with $V$. nonalfalfae, other DE miRNAs differ between the two cultivars.

In $V$. nonalfalfae-inoculated root samples of the susceptible cultivar Celeia, we identified seven differentially expressed miRNAs, five of which belong to four different miRNA families (MIR159, MIR828, MIR477 and MIR167) and two novel miRNAs (miRNA363/miRNA1427 and miRNA-898/miRNA-2452) belong to two different novel miRNA families. A significant upregulation was detected for hlu-miR159c-d $\left(\log _{2} \mathrm{FC}=1.2\right)$ and a significant downregulation for hlu-miR828a-b $\left(\log _{2} \mathrm{FC}=-3.5\right)$, hlu-miR477 $\left(\log _{2} \mathrm{FC}=-2.1\right)$ and two members of MIR167, i.e., hlu-miR167f $\left(\log _{2} \mathrm{FC}=-2.1\right)$ and hlu-miR167a-d $\left(\log _{2} \mathrm{FC}=-1.5\right)$. Additionally, a significant downregulation was detected for two novel miRNAs, namely miRNA-363/miRNA-1427 $\left(\log _{2} \mathrm{FC}=-2.6\right)$ and miRNA-898/miRNA$2452\left(\log _{2} \mathrm{FC}=-2.5\right)$ (Figure $2 \mathrm{a}$ and Table S3).
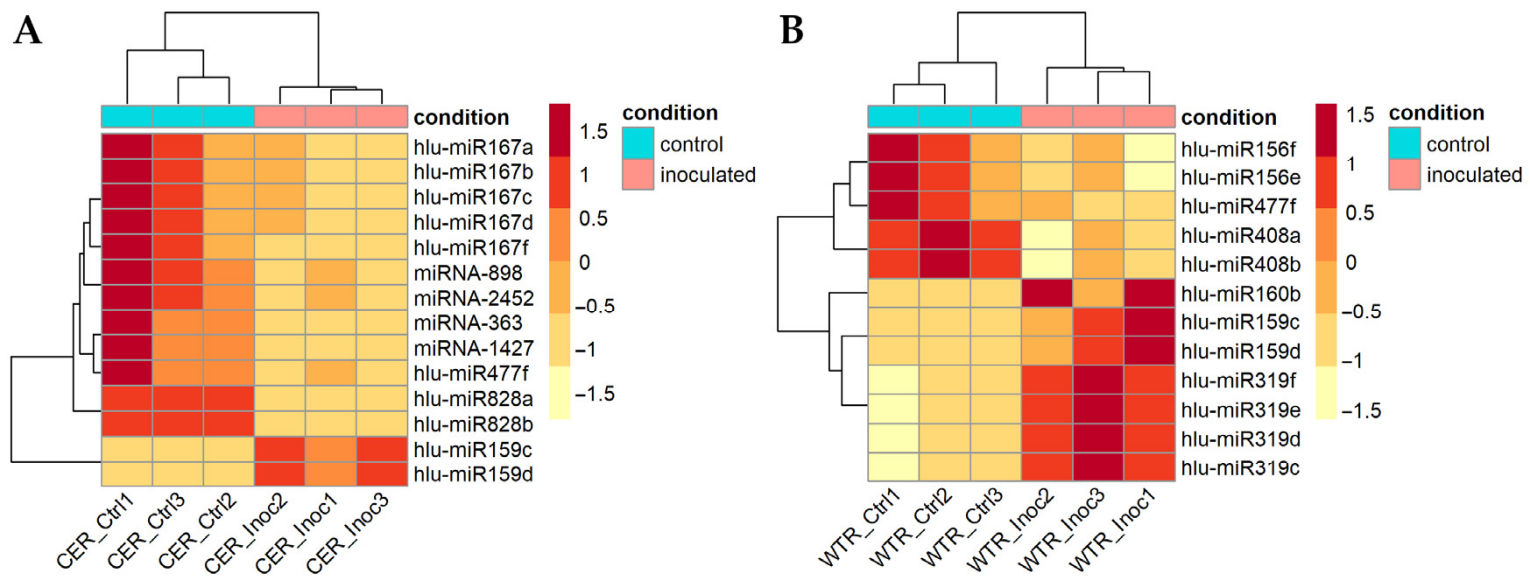

Figure 2. Heat map of differentially expressed miRNAs between $V$. nonalfalfae-inoculated (Inoc) and control (Ctrl) root samples (three biological replicates) of (A) the susceptible cultivar Celeia (CER) and (B) the resistant cultivar Wye Target (WTR). The colour scale represents the $\mathrm{z}$-score scaled by row of the normalized read counts. Clustering was performed using the Euclidean distance measure and complete clustering method.

In $V$. nonalfalfae-inoculated root samples of the resistant cultivar Wye Target, we identified six known differentially expressed miRNAs that belong to six different miRNA families (MIR408, MIR477, MIR156, MIR160, MIR319 and MIR159). Three miRNAs were upregulated, i.e., hlu-miR160b $\left(\log _{2} \mathrm{FC}=1.2\right)$, hlu-miR319c-f $\left(\log _{2} \mathrm{FC}=0.9\right)$ and hlu-miR159c-d $\left(\log _{2} \mathrm{FC}=1\right)$, while hlu-miR408a-b $\left(\log _{2} \mathrm{FC}=-1.4\right)$, hlu-miR477f $\left(\log _{2} \mathrm{FC}=-1.6\right)$ and hlu-miRR156e-f $\left(\log _{2} \mathrm{FC}=-0.65\right)$ were downregulated (Figure $2 \mathrm{~b}$ and Table S4). 


\subsection{Differentially Expressed MiRNAs between Susceptible and Resistant Hop Cultivars}

Comparing miRNA response to $V$. nonalfalfae inoculation between susceptible and resistant hop cultivars, the resistant cultivar WT shows higher expression of hlu-miR167a-d and hlu-miR167f $\left(\log _{2} \mathrm{FC}=1.3\right.$, respectively), hlu-miR828a-b $\left(\log _{2} \mathrm{FC}=2.5\right)$ and a novel miRNA family containing miRNA-363 and miRNA-1427 $\left(\log _{2} \mathrm{FC}=2.4\right)$, and are thus clustered together (Figure 3). On the other hand, hlu-miR390a $\left(\log _{2} \mathrm{FC}=-2.2\right)$, hlu-miR169a-d $\left(\log _{2} \mathrm{FC}=-2.2\right)$, hlu-miR169m-n $\left(\log _{2} \mathrm{FC}=-1.9\right)$, hlu-miR164a-c $\left(\log _{2} \mathrm{FC}=-1.4\right)$, hlumiR408a-b $\left(\log _{2} \mathrm{FC}=-3.9\right)$, hlu-miR171g-h $\left(\log _{2} \mathrm{FC}=-1.3\right)$ and a novel miRNA-617 $\left(\log _{2} \mathrm{FC}=-6.3\right)$ shows lower expression in response to inoculation with $V$. nonalfalfae (Figure 3 and Table S5).

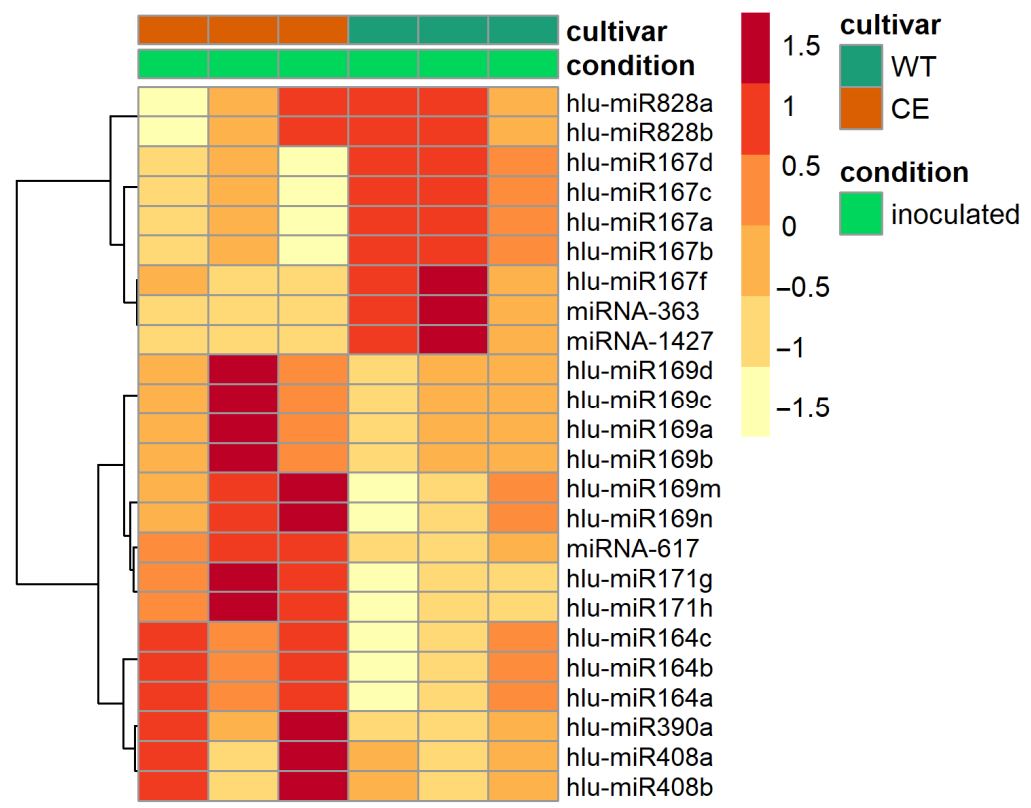

Figure 3. Heat map of differentially expressed miRNAs between the susceptible cultivar Celeia (CE) and the resistant cultivar Wye Target (WT) in response to inoculation with $V$. nonalfalfae. Clustering was performed using the Euclidean distance measure and Ward clustering method. The colour scale represents the z-score scaled by row of the control-normalized read counts.

2.5. MiRNA Target Prediction, GO Analysis of miRNA Targets and Protein-Protein Interaction Network Analysis

In silico psRNATarget analysis [37] of miRNA target transcripts was performed for differentially expressed miRNAs of susceptible and resistant hop cultivars and revealed 49 transcripts that are potential targets (Table 2 and Table S8). For a single miRNA, psRNATarget identified from one to up to nine different target transcripts and one transcript is targeted by more than one DE miRNA (Table 2). Transcription factor GAMYB (W9QVM8) is targeted by two different miRNAs; hlu-miR159c-d, which is 1.28 - and 0.95 -fold $\left(\log _{2}\right)$ upregulated in the susceptible and resistant cultivar, respectively, and by hlu-miR319c-f, which is 0.85 -fold $\left(\log _{2}\right)$ upregulated in the resistant hop cultivar. Most of the identified targets of DE miRNAs were found to encode transcription factors or transcriptional regulators. For example, auxin response factor (ARF) is targeted by hlu-miR160b, which is upregulated in the resistant cultivar. Moreover, some targets encode proteins involved in effector-triggered immunity, e.g., wall-associated receptor kinase (a target of novel miRNA617) [38], or are involved in metabolic pathways (polyphenol oxidase; a target of novel miRNA-363/miRNA-1427) [39]. 
Table 2. Differentially expressed miRNAs in response to inoculation with $V$. nonalfalfae and their target transcripts in hops.

\begin{tabular}{|c|c|c|c|c|}
\hline \multirow{2}{*}{ miRNA } & \multicolumn{2}{|c|}{$\log _{2} \mathrm{FC} ;$ adj. $p \leq 0.1$} & \multirow{2}{*}{$\begin{array}{l}\text { Sig. Interaction; } \\
p \leq 0.05\end{array}$} & \multirow{2}{*}{ Target Transcript (Orthologue ID *) } \\
\hline & & & & \\
\hline hlu-miR156e-f & NS & -0.65 & NS & 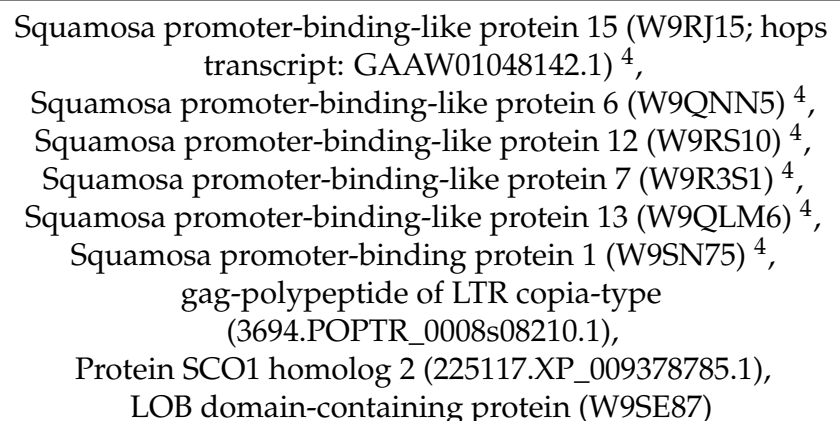 \\
\hline & & & & Putative anion transporter 3 (W9RGS2) ${ }^{1,3}$ \\
\hline hlu-miR159c-d & 1.28 & 0.95 & NS & $\begin{array}{c}\text { Transcription factor GAMYB (W9QVM8) })^{2,4}, \\
\text { Integrase catalytic domain-containing protein } \\
\text { (A0A087HSL5) }{ }^{1,2,3,4}\end{array}$ \\
\hline & & & & $\begin{array}{l}\text { Acetyltransferase At3g50280-like (M5VT21) }{ }^{2,4}, \\
\text { Transcription factor MYB51-like (102107.XP_008226955.1), } \\
\text { Cytochrome p450 (57918.XP_004291627.1) }\end{array}$ \\
\hline hlu-miR160b & NS & 1.21 & NS & $\begin{array}{c}\text { Auxin response factor (A0A061FPV2) } \\
\text { Auxin response factor (W9QUH2) } \\
\text { Auxin response factor (W9S7Q7) } \\
\text { 3,4 }\end{array}$ \\
\hline hlu-miR164b & NS & NS & -1.4 & $\begin{array}{l}\text { NAC domain-containing protein } 100 \text { (W9QTW9), } \\
\text { NAC domain-containing protein (W9QCM5; hops transcript: } \\
\text { GAAW01060518.1) }\end{array}$ \\
\hline hlu-miR167a-d & -1.5 & NS & 1.35 & $\begin{array}{l}\text { Mediator of RNA polymerase II transcription subunit } \\
\text { (102107.XP_008227322.1) }\end{array}$ \\
\hline hlu-miR167f & -2.1 & NS & 1.34 & $\begin{array}{l}\text { Mediator of RNA polymerase II transcription subunit } \\
\text { (102107.XP_008227322.1) }\end{array}$ \\
\hline hlu-miR169a-d & NS & NS & -2.2 & $\begin{array}{l}\text { Nuclear transcription factor Y subunit A-8 (W9QJW4), } \\
\text { ATPase (W9WDM0), } \\
\text { Nuclear transcription factor Y subunit A-1 (W9RR19), } \\
\text { Nuclear transcription factor Y subunit A-10 (W9SK30) } \\
\text { Nuclear transcription factor Y subunit A-10 (W9SK30), }\end{array}$ \\
\hline hlu-miR169m-n & NS & NS & -1.95 & $\begin{array}{l}\text { Nuclear transcription factor Y subunit A-1 (W9RR19), } \\
\text { TATA-binding protein-associated factor (W9R0N8), } \\
\text { Exo84_C domain-containing protein (W9SE15) }\end{array}$ \\
\hline hlu-miR171g-h & NS & NS & -1.3 & $\begin{array}{c}\text { Scarecrow-like protein } 22 \text { (102107.XP_008238556.1), } \\
\text { Scarecrow-like protein 6-like (57918.XP_004306953.1), } \\
\text { GRAS domain-containing protein (A0A251QFM0; hop } \\
\text { transcript: GAAW01082848.1), }\end{array}$ \\
\hline hlu-miR319c-f & NS & 0.85 & NS & $\begin{array}{l}\text { NAD(P)-bd_dom domain-containing protein (M5VYK9) } \\
\text { Transcription factor GAMYB (W9QVM8) } 2,4 \\
\text { Teosinte branched 1, putative isoform } 1 \text { (A0A061GDP3) }\end{array}$ \\
\hline hlu-miR390a & NS & NS & -2.2 & $\begin{array}{l}\text { Regulation of response to stimulus } \\
(218851 . \text { Aquca_1504_00001.1) } \\
\text { ARM repeat superfamily protein isoform 1 (A0A061G6D5) } \\
\text { Mitochondrial protein (3827.XP_004514187.1) } \\
\text { Cation-transporting ATPase (W9RQ63) }\end{array}$ \\
\hline hlu-miR408a-b & NS & -1.41 & -3.9 & Long chain acyl-CoA synthetase 8 (W9QFE0) ${ }^{4}$ \\
\hline hlu-miR828a-b & -3.50 & NS & 2.5 & $\begin{array}{l}\text { Serine/threonine-protein phosphatase (W9QNS5) }{ }^{2} \text {, } \\
\text { Nuclear pore membrane glycoprotein (W9S8T7), } \\
\text { RNA pol II transcription regulator recruiting } \\
\text { activity-ATMYB5 (3649.evm.model.supercontig_96.57), } \\
\text { 3-hydroxyisobutyryl-CoA hydrolase (A0A087GEK8) }\end{array}$ \\
\hline
\end{tabular}


Table 2. Cont.

\begin{tabular}{|c|c|c|c|c|}
\hline \multirow{2}{*}{ miRNA } & \multicolumn{2}{|c|}{$\log _{2} \mathrm{FC} ;$ adj. $p \leq 0.1$} & \multirow{2}{*}{$\begin{array}{l}\text { Sig. Interaction; } \\
p \leq 0.05\end{array}$} & \multirow{2}{*}{ Target Transcript (Orthologue ID *) } \\
\hline & $\mathrm{DE}$ in $\mathrm{CE}$ & DE in WT & & \\
\hline $\begin{array}{l}\text { miRNA-363 } \\
\text { miRNA-1427 }\end{array}$ & -2.61 & NS & 2.4 & $\begin{array}{l}\text { Polyphenol oxidase (W9S222) }{ }^{1,2} \text {, } \\
\text { ER lumen retaining receptor family (F6HCQ4) }{ }^{1,2} \\
\text { Dynamin-related protein } 4 \mathrm{C}(\mathrm{W} 9 \mathrm{QQY9})^{2}\end{array}$ \\
\hline $\begin{array}{l}\text { miRNA-898 } \\
\text { miRNA-2452 }\end{array}$ & -2.49 & NS & NS & $\begin{array}{l}\text { L-threonine ammonia-lyase activity } \\
\qquad\left(161934 . X P \_010694863.1\right),\end{array}$ \\
\hline miRNA-617 & NS & NS & -6.35 & $\begin{array}{l}\text { Wall-associated receptor kinase (F6GSN9), } \\
\text { DIS3-like exonuclease } 2 \text { (W9QVR2) }\end{array}$ \\
\hline
\end{tabular}

Sig. interaction is the difference between the condition effect for the resistant cultivar compared to the condition effect for the susceptible cultivar. * Orthologue ID is either UniProt (Entry) or STRING identifier. NS = not significant. ${ }^{1,2}$ Significant genes identified in topGO analysis of biological process and molecular function ontologies, respectively, performed with targets of differentially expressed miRNAs in the susceptible cultivar. 3,4 Significant genes identified in topGO analysis of a biological process and molecular function ontologies, respectively, performed with targets of differentially expressed miRNAs in the resistant cultivar.

Gene ontology analysis (GO) showed that the targeted transcripts of the susceptible cultivar are enriched for twenty-one GO terms of biological processes with six targets that significantly contribute to enriched GO terms (significant targets), while seven enriched GO terms with eight significant targets were identified for molecular functions (Tables 2 and S6). In the resistant cultivar, GO analysis revealed fifty-one enriched GO terms with five significant targets in the biological process ontology and six enriched GO terms with sixteen significant targets in the molecular function ontology (Tables 2 and S7).

It is noteworthy that susceptible and resistant cultivars alter the expression of different miRNAs in response to inoculation with $V$. nonalfalfae and, therefore, the biological processes and molecular functions of their targets are expected to differ. In the susceptible cultivar, enriched biological processes of the DE miRNA targets include transportation and localization, i.e., protein retention in ER lumen, maintenance of protein localization, vesiclemediated transport and cytosolic transport, and pigment biosynthetic process. Enriched molecular functions of the DE miRNA targets are ER retention sequence binding, signal sequence binding and catechol oxidase activity (Table S6). Targets of DE miRNAs in the resistant cultivar are involved in the auxin-activated signalling pathway, various regulatory processes, e.g., regulation of DNA-templated transcription, macromolecule biosynthetic process, nitrogen compound metabolic process and aromatic compound biosynthetic process, etc., and have various binding functions (Table S7).

The protein-protein interaction network of miRNA targets and their interactors comprises a total of 194 nodes (proteins or miRNAs) and 475 edges (connections), with each node connected to an average of 5.4 neighbours (interactors). Network analysis of proteinprotein interactions of miRNA targets and their interactors revealed that the local network clusters comprising the targets of downregulated miRNAs in the susceptible hop cultivar are enriched in terms related to the TFIIS helical bundle-like domain, annexin, catechol oxidase activity, glycoside transport, laccase, Rer1 family, protein processing in ER, ERGolgi transport, MYB-like domain, macromolecule metabolic process and biosynthesis of secondary metabolites (Figure 4). On the other hand, the upregulated miRNA local network clusters are enriched in terms related to cytochrome, transport, oxidative phosphorylation, ATP synthase, and trichome birefringence-like family. The latter three terms are also enriched in the upregulated miRNA local network clusters of the resistant hop cultivar. In addition, in the resistant hop cultivar, local network clusters with miRNA targets of upregulated miRNAs are enriched in terms related to the RNA metabolic process, auxin-activated signalling pathway, phosphoglycolate phosphatase-like, QLQ domain, PAR1 protein, and nucleotide sugar transporter (Figure 4). Local network clusters with targets of downregulated miRNAs in the resistant cultivar are enriched for terms related to the RNA metabolic process, BZR family, axillary shoot meristem initiation (tissue develop- 
ment) and fatty acid metabolic process. Moreover, local network clusters with significantly lower miRNA expression in the resistant cultivar compared to the susceptible hop cultivar after $V$. nonalfalfae inoculation, showed enrichment in terms related to leucine-rich repeat (Figure 4).

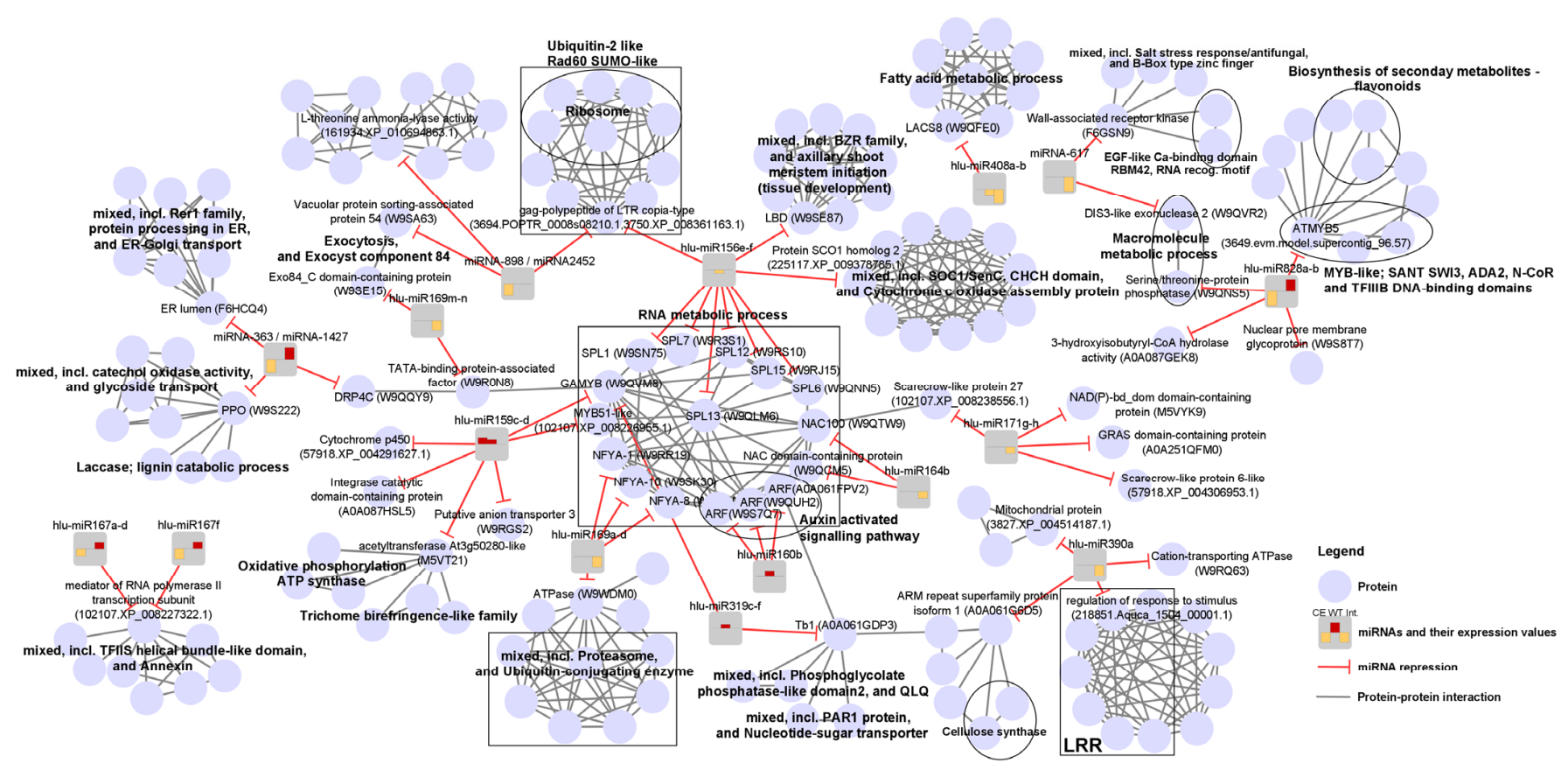

Figure 4. Protein-protein interaction network of miRNAs and their targets with interactors. Blue circles represent proteins and interactions between them (grey lines), and grey round rectangles with bar plot represent miRNAs repressing their targets (red lines with T-shaped arrowhead indicating target repression). The first and second bars of the miRNA bar plot represent miRNA expression levels (red, upregulated; orange, downregulated) determined by DESeq2 analysis for the sensitive hop cultivar Celeia (CE) and the resistant hop cultivar Wye Target (WT), respectively, upon inoculation with $V$. nonalfalfae. The third bar represents the interaction (Int.), defined as the difference between the condition effect for the resistant cultivar compared to the condition effect for the susceptible cultivar. Enriched terms of local network clusters obtained by the STRING Enrichment analysis are shown in bold. miRNA target names have either UniProt (Entry) or STRING identifier. ARF, Auxin response factor; NF-YA, Nuclear transcription factor Y subunit A; SPL, Squamosa promoterbinding-like protein; DRP4C, Dynamin-related protein 4C; LBD-LOB domain-containing protein; LRR, Leucine-rich repeat proteins; Tb1, Teosinte branched 1; LACS8, Long chain acyl-CoA synthetase 8; ER, endoplasmic reticulum.

\section{Discussion}

We sequenced 12 sRNA libraries from three control and three $V$. nonalfalfae-inoculated roots of the susceptible cultivar Celeia or the resistant cultivar Wye Target. Using the miR-PREFeR miRNA prediction pipeline, we identified 56 mature miRNAs belonging to 30 known plant miRNA families and 35 novel miRNA families represented by a different number of members.

In our study, a significant upregulation in response to inoculation with $V$. nonalfalfae was observed for hlu-miR159c-d in both hop cultivars. Similarly, as reported, miR159 was upregulated in $P$. beijingensis inoculated with D. gregaria [19], P. trichocarpa inoculated with Botryosphaeria dothidea [40] and in Triticum aestivum during Puccinia graminis f.sp. tritici infection [41]. The main role of the MIR159 family is the regulation of GAMYB or GAMYB-like transcription factors that possess highly conserved binding sites for miR159 [42]. Previous studies have shown that miR159 represses primary root growth by inhibiting MYB33, MYB65 and MYB101 [43]. In silico miRNA target prediction revealed that hlu-miR159c-d (upregulated in both cultivars) and hlu-miR319c-f (upregulated in the resistant cultivar) bind to transcripts of GAMYB but also have other distinct targets. Moreover, hlu-miR319c-f targets transcripts of the hops' teosinte branched 1 (tb1) (A0A061GDP3), which belongs to 
the TCP transcription factor family. While both miRNAs regulate MYB TFs in Arabidopsis, miR319 acts predominantly on transcription factors of the TCP family and, to a lesser extent, on the expression of $M Y B$ since the expression levels and domain of miR319 limit its regulation of $M Y B$, while the sequence of miR159 prevents binding to TCP transcripts $[44,45]$. The latter is also observed in our study as we did not detect the binding site of miR159 within TCP transcripts. Teosinte branched 1 acts as a negative regulator of lateral branching [46,47], therefore its downregulation by upregulated hlu-miR319c-f in the resistant hop cultivar may lead to the secondary growth and branching of roots that may in turn help to sustain the vitality of the resistant cultivar during infection with $V$. nonalfalfae.

A significant downregulation was observed in our study for hlu-miR156e-f in the resistant hop cultivar, and in silico target prediction showed that it targets transcripts of six different Squamosa promoter-binding-like protein genes (SPL), SCO1 homolog 2 and LATERAL ORGAN BOUNDARIES (LOB) domain-containing protein (LBD). The interaction between hops' miR156 and SPB15 transcripts was previously confirmed by $5^{\prime}$ RLM-RACE analysis [48]. Furthermore, Bhogale et al. [49] validated the interaction between miR156 and StSPL3, StSPL6, StSPL9, StSPL13 in Solanum tuberosum ssp. andigena and observed that miR156 can be transported through the plants via the phloem. Proteins from the SPB family are thought to be transcriptional activators and have roles in leaf development, vegetative phase change, flower and fruit development, plant architecture, shoot maturation, gibberellin signalling and response to fungal toxin [50,51].

Another interesting target of hlu-miR156e-f is the transcript of the LOB domaincontaining protein (W9SE87). The latter protein family is involved in secondary growth and the development of xylem and phloem tissue [52] through a positive feedback loop that promotes the expression of the NAC domain-containing protein 30 (NACO30)/vascular-related NAC domain protein7 (VND7), which regulates genes associated with the differentiation of tracheary elements in Arabidopsis, e.g., genes involved in secondary wall biosynthesis, cell wall modifications such as xylan accumulation and programmed cell death $[53,54]$. Vascular-related NAC domain7 TF plays an important role in the response to infection with $V$. longisporum in Arabidopsis, as it induces de novo formation of functional xylem elements from bundle sheath cells, which subsequently leads to vein clearing and xylem hyperplasia within the vasculature of the roots, as well as to enhanced drought tolerance [55]. This may suggest that downregulation of hlu-miR156e-f and the resulting expression of SPL and LBD in the resistant hop cultivar modulates root development and vascular tissue processes in roots, which could contribute to a successful defence response after inoculation with V. nonalfalfae.

In response to inoculation with $V$. nonalfalfae, the resistant cultivar showed significantly lower expression of hlu-miR164b, which targets transcripts of NAC domain-containing proteins. The hlu-miR164b cleavage site within the transcript of the hops' NAC domaincontaining protein was confirmed by $5^{\prime}$ RLM-RACE analysis performed by Mishra et al. [48]. Hu et al. [56] observed a significant decrease of ghr-miR164 in the response of cotton plants to infection with $V$. dahliae. Additionally, the researchers showed that ghr-miR164 directly cleaves the mRNA of GhNAC100, and silencing of ghr-miR164 leads to increased GhNAC100 expression, which in turn increases plant resistance to the fungus. The decrease of miR164 was also observed in Oryza sativa upon infection with Magnaporthe oryzae strain Guy11, and rice plants with the dysfunctional miR164a/OsNAC60 regulatory module developed a significant susceptibility to infection with Guy11 [57]. Auxin-induced expression of miR164 in wild-type Arabidopsis plants resulted in decreased levels of the NAC1 transcripts and reduced lateral root emergence. Additionally, Arabidopsis mir164a and mir164b mutants that express less miR164 show higher expression of $N A C 1$ and have abundant lateral roots. This evidence may indicate that the auxin-miR164-NAC1 regulation provides a homeostatic mechanism that modulates auxin signalling during lateral root development [58].

Hormone signalling pathways modulate plant responses to biotic stress [59] and may be involved in a trade-off between primary growth and response mechanisms of the resistant hop cultivar during Verticillium wilt pathogenesis. Hlu-miR160b, which is 
significantly upregulated in inoculated compared to control root samples of the resistant hop cultivar, is predicted to target transcripts of auxin response factors $(A R F)$, DNA-binding proteins that bind to a specific sequence in promoters of auxin-responsive genes [60]. Upregulation of miR160 and its regulation of $A R F$ s has also been demonstrated during the pathogenesis of stem canker disease in P. trichocarpa [40] and in potato, where it targets StARF10, which binds to the promoter in the StGH3.6 gene, a mediator of salicylic acidauxin cross-talk, and is thus associated with local defence and systemic acquired resistance to $P$. infestans [14]. In A. thaliana, miR160 controls root cap formation by regulating the expression of ARF10 and ARF16. Disturbed miR160-directed regulation of ARF16 resulted in reduced fertility and fewer lateral roots [61]. In addition, researchers observed defects in root growth in Arabidopsis plants expressing an miR160-resistant version of ARF17 [62].

The downregulation of hlu-miR156e-f, and the upregulation of hlu-miR160b and hlu-miR319c-f in inoculated root samples compared to the controls of the resistant hop cultivar, and the significantly lower expression of hlu-miR164b in the resistant hop cultivar compared to the susceptible hop cultivar, may indicate the role of these miRNAs in modulating hormone signalling and the processes of root growth, branching and vascular tissue development in the resistant hop cultivar during infection with $V$. nonalfalfae.

Compared to the susceptible hop cultivar, the resistant cultivar showed significantly lower expression of MIR169 and, by in silico analysis of miRNA targets, we identified nuclear transcription factor Y (NF-YA) as a target of miR169. Similarly, Li et al. [63] observed a higher expression of miR169 in the susceptible O. sativa cultivar but not in the resistant cultivar when infected with $M$. oryzae. In rice, miR169 suppresses the expression of NFYA genes and, thus, acts as a negative regulator in rice immunity against blast fungus M. oryzae, since the transgenic lines that overexpress miR169 become hypersusceptible to $M$. oryzae due to the reduced expression of defence-related genes [63]. A significantly lower expression of miR169 in the resistant hop cultivar might, thus, contribute to hop resistance. In addition, miR169 regulates NF-YA2 and NF-YA10 genes involved in the control of primary root growth [64], further suggesting that processes of root growth are pronounced in the resistant hop cultivar inoculated with $V$. nonalfalfae.

The resistant hop cultivar inoculated with $V$. nonalfalfae also showed significantly lower expression of hlu-miR390a compared to the susceptible hop cultivar. One of its targets is involved in the regulation of response to stimuli and encodes proteins with successive leucine-rich repeat motifs. This hop protein may belong to the class of Toll-like receptors that bind pathogen- and danger-associated molecular patterns [65] and may be involved in the defence response to $V$. nonalfalfae infection. Similarly, after inoculation, the resistant cultivar showed significantly lower expression of the novel miRNA-617 than the susceptible hop cultivar. The cleavage site of miRNA-617 was predicted in transcripts of wall-associated receptor kinase from the protein family of receptor-like kinases (RLK), which are involved in the recognition of pathogens and signal transduction during pathogen attack [38].

In the resistant cultivar, we observed a downregulation of hlu-miR408a-b and a significantly lower expression of the latter in response to inoculation with $V$. nonalfalfae compared to the susceptible hop cultivar. Yin et al. [66] observed an increased expression of miR408 in Arabidopsis plants that overexpressed the effector protein SR1-a of Puccinia graminis f. sp. tritici (Pgt), but the increase was not significantly higher in wheat leaves. In our study, the binding site of miR408 was found in transcripts of the long chain acylcoenzyme synthetase 8 (LACS8) that is associated with fatty acid metabolic process, and LACS1 and LACS2 that are involved in cutin biosynthetic process. In A. thaliana, the long chain acyl-coenzyme synthetase activates C16 or C18 fatty acids, which represent a substrate for cutin and wax [67]. The downregulation of miR408 may modulate the biosynthetic pathways of cutin and wax, which could lead to the accumulation of these compounds in the roots of the resistant hop cultivar when inoculated with $V$. nonalfalfae. Moreover, Progar et al. [28] observed enriched biological processes related to cell wall biogenesis and cutin biosynthesis in the transcriptomic study of interactions between $V$. nonalfalfae and resistant hop cultivar Wye Target. 
In the susceptible hop cultivar inoculated with $V$. nonalfalfae, we observed a significant downregulation of hlu-miR167a-d, hlu-miR167f and hlu-miR828a-b, and two novel miRNAs, i.e., miRNA-363/miRNA-1427 and miRNA-898/miRNA-2452. All aforementioned miRNAs, except miRNA-898/miRNA-2452, had significantly higher expression in the resistant cultivar in response to inoculation with $V$. nonalfalfae.

Recent studies showed that miR828 positively regulates phenylpropanoid biosynthesis either by direct cleavage of $M Y B$ transcripts or by cleaving the transcripts of trans-acting siRNA gene 4 (TAS4), which results in the production of ta-siRNAs that silence the expression of the $M Y B$ gene [68,69]. In our study, cleavage sites of hlu-miR828a-b were predicted in transcripts of serine/threonine-protein phosphatase, 3-hydroxyisobutyrylCoA hydrolase and transcripts of genes encoding proteins with RNA pol II transcription regulator recruiting activity $(A T M Y B 5)$. The latter protein contains DNA-binding domains of MYB-related proteins or SANT domain, and forms a complex with a basic helix-loophelix protein and the WD40 protein family that is involved in regulation of the flavonoid pathway [70]. These transcripts significantly contribute to GO-enriched terms of molecular functions and biological processes related to peptide binding and pigment biosynthesis in the susceptible hop cultivar and are also present in the local network cluster enriched for biosynthesis of secondary metabolites.

In contrast to the resistant hop cultivar, in which we may observe pronounced miRNAmediated regulation of processes related to root growth and vascular tissue development following inoculation with $V$. nonalfalfae, miRNA regulation in the susceptible hop cultivar mediates transcriptional reprogramming leading to changes in various metabolic processes.

Cleavage sites of the novel miRNA-363/miRNA-1427 were identified in transcripts of polyphenol oxidase, a protein from the family of the ER lumen retaining receptors and in dynamin-related protein 4C. In previous studies, novel miRNAs targeting polyphenol oxidase were identified in P. trichocarpa [18], Salvia miltiorrhiza [39], Solanum tuberosum [71] and Vitis vinifera [72]; however, they differ in sequence from miRNA-363/miRNA-1427 identified in our study. The other two potential targets, the protein of the ER lumen retaining receptor family and dynamin-related protein $4 \mathrm{C}$, are both involved in cellular localization or transport and have contributed significantly to the enrichment of these biological processes and molecular functions. Additionally, novel miRNA miRNA-898/miRNA-2452 potentially targets transcripts of vacuolar protein sorting-associated protein, which is involved in protein transport between endosomes and the trans-Golgi network [73] and, in the susceptible hop cultivar, is significant in the GO biological process terms associated with transport.

\section{Materials and Methods}

\subsection{Inoculation of Hop Plants}

Hop plants of the susceptible cultivar Celeia and the resistant cultivar Wye Target were provided by Slovenian Institute for Hop Research and Brewing. Hop plants were vegetatively propagated as softwood cuttings in a greenhouse or as dormant cuttings from the rootstock. One-year-old rooted cuttings were used in the experiment. The plants were inoculated by root dipping method using the well-established protocol proposed by Flajsman et al. [74]. Briefly, the roots of 3 biological replicates of one-year-old rooted cuttings of each cultivar were immersed for $10 \mathrm{~min}$ in a suspension containing conidia of the highly virulent strain of $V$. nonalfalfae (PV1, isolate T2) $\left(5 \times 10^{6}\right.$ conidia/mL), and the roots of 3 control plants of each cultivar were mock-inoculated using sterile water. Artificially inoculated and mock-inoculated (control) whole root tissues were sampled $24 \mathrm{~h}$ after inoculation. Roots were cut from stems at the first node, washed, freeze-dried with liquid nitrogen, and ground to a fine powder with mortars and pestles. Following grinding, the samples were stored at $-80^{\circ} \mathrm{C}$ until total RNA and small RNAs were isolated. 


\subsection{Small RNA Isolation, Library Construction and Sequencing}

Small RNAs were isolated from $100 \mathrm{mg}$ root tissue of both cultivars in three $V$. nonalfalfae-inoculated and three control replicates, using mirVana ${ }^{\mathrm{TM}}$ miRNA Isolation Kit (Waltham, MA, USA) according to manufacturer's instructions for the enrichment of small RNAs. The quantity and quality of the small RNA-enriched sample and miRNA fraction were assessed with Agilent ${ }^{\circledR} 2100$ Bioanalyzer ${ }^{\circledR}$ instrument (Agilent Technologies, Inc., Santa Clara, CA, USA) using Bioanalyzer Agilent ${ }^{\circledR}$ Small RNA Kit, following the manufacturer's instruction. Thus, we determined the input amount of small RNAs, to construct three control and three $V$. nonalfalfae-inoculated small RNA libraries for each cultivar. Small RNA libraries were constructed using the Ion Total RNA-Seq Kit v2 and Ion Xpress ${ }^{\mathrm{TM}}$ RNA-Seq Barcode 1-16 Kit following the manufacturer's instructions. Briefly, adaptors were hybridized and ligated to small RNAs, and the reverse transcription was performed. Afterwards, purification and size-selection were performed using magnetic beads to obtain only miRNAs and other small RNAs to which barcodes were added through PCR amplification. The yield and size distribution of amplified cDNA libraries were assessed with Agilent ${ }^{\circledR} 2100$ Bioanalyzer ${ }^{\circledR}$ instrument (Agilent Technologies, Inc., Santa Clara, CA, USA) and Agilent ${ }^{\circledR}$ High Sensitivity DNA Kit to pool equimolar barcoded libraries of each cultivar separately. Three inoculated and three mock-inoculated barcoded libraries of susceptible or resistant cultivars were pooled in equimolar concentration and prepared for sequencing according to the manufacturer's instructions, accompanying Ion PI ${ }^{\mathrm{TM}} \mathrm{Hi}-\mathrm{Q}^{\mathrm{TM}} \mathrm{OT} 2200 \mathrm{Kit}$ and Ion PI ${ }^{\mathrm{TM}}$ $\mathrm{Hi}-\mathrm{Q}^{\mathrm{TM}}$ Sequencing $200 \mathrm{Kit}$. Both prepared samples were sequenced on the Ion Proton ${ }^{\mathrm{TM}}$ System (Waltham, MA, USA). The raw sequencing data were deposited to the SRA archive (BioProject ID PRJNA665133).

\subsection{Prediction, Identification and Differential Expression Analysis of miRNAs in Hops}

Prior to bioinformatics analysis, barcodes, adapters and low-quality raw sequence reads were removed using FastQC software (https: / / www.bioinformatics.babraham.ac. uk/projects/fastqc/; accessed on 30 August 2021) and high-quality sequencing reads were used for further analysis. Briefly, FASTA files containing sRNA-seq reads were preprocessed using scripts provided by miR-PREFeR pipeline [32] and the reads were aligned with Bowtie [75], disallowing mismatches, against hop draft genome sequences obtained from HopBase [30,31]. Afterwards, alignment files of processed RNA-Seq reads were used to predict hop miRNAs using a miR-PREFeR pipeline with parameters set according to criteria for plant miRNA annotation [76].

To identify known miRNA families in hops, predicted mature or precursor miRNA sequences were aligned with Bowtie2 [77] against mature or precursor sequences in the microRNA database (miRBase Release 22.1) [33]. Additionally, predicted precursor miRNA sequences were aligned against RNAcentral, a non-coding RNA sequence database [34].

Minimum folding energy (MFE) of secondary structures of predicted precursor miRNAs was calculated using RNAfold tool [78] and used to calculate adjusted minimal folding free energy (AMFE), which enables indirect comparison of MFEs among predicted known and novel pre-miRNAs [79].

sRNA counts of predicted mature miRNAs provided by miR-PREFeR output were subjected for differential expression analysis in R version 3.5.1 [80]. Count matrices containing read counts of control and inoculated samples were constructed for susceptible and resistant cultivars, respectively. Prior to differential expression analysis, predicted mature miRNAs with less than 100 read counts in control and inoculated samples were discarded. The differential expression analysis of predicted mature miRNAs was performed with DESeq2 [81]. Predicted miRNAs with FDR corrected $p$-value $<0.1$ were treated as significantly differentially expressed between inoculated and control samples. Filtering according to the $\log _{2}$ fold change parameter was not applied because we wanted to detect low but significant changes in the expression of miRNAs.

To test whether the treatment effect differs across cultivars, the interaction term was added to the model in DESeq2 and the entire read-count matrix containing mature 
miRNAs with more than 200 read counts in all samples was used in the differential expression analysis. Predicted mature miRNAs with $p$-value $\leq 0.05$ were considered as differentially expressed.

Furthermore, predicted precursors of novel miRNAs (novel pre-miRNAs) and known pre-miRNAs from miRBase were clustered using CD-HIT-EST [36] with a global sequence identity threshold 0.8 . Predicted novel pre-miRNAs clustered with annotated pre-miRNAs were grouped into corresponding known miRNA families and predicted novel pre-miRNAs that did not show similarity were categorized as novel miRNA families.

\subsection{In Silico Prediction of MiRNA Targets of Differentially Expressed MiRNAs, Gene Ontology and Protein-Protein Network Analyses}

MicroRNA target analysis was performed on-line using psRNATarget Analysis Server (2017 Update) [37]. Mature miRNA sequences of differentially expressed miRNAs were used in in silico miRNA target prediction analysis. The targets of differentially expressed miRNA of susceptible and resistant hop cultivars were predicted in annotated hop transcriptome [82] with the following parameters: max expectation cut-off: 2.5 , seed region: 2-13, number of mismatches allowed in seed region: 2, range of mismatch disable slicing: 9-11, HSP length for scoring: 19, penalty for GU pair: 0.5, penalty for other mismatches: 1.0, allowing bulge on target, penalty for opening gap: 2.0, penalty for extending gap: 0.5 , weight for seed region: 1.5 , calculating UPE around the target site (target accessibility analysis): $17 \mathrm{nt}$ upstream and $13 \mathrm{nt}$ downstream. Afterwards, gene ontology (GO) analysis was performed using $\mathrm{R}$ package topGO (version 2.40.0) [83] on targets of differentially expressed miRNAs in order to identify overrepresented/enriched GO terms and significant miRNA targets belonging to enriched GO terms. Classical enrichment analysis was performed with Fisher's statistical test ( $p$-value $\leq 0.05)$. To obtain a broader picture of the functions of hops miRNA targets, a protein-protein interaction network was constructed using Cytoscape [84]. For targets representing a single node, 10 additional interactors were sought in the string database with a cut-off of 0.8 confidence (score). For each local network cluster comprising the miRNA targets and its interactors, an enrichment analysis was performed using the built-in stringApp [85].

\section{Conclusions}

Hops have become an increasingly important crop agronomically, mainly due to their use in the brewing industry and, more recently, in the pharmaceutical industry. In our study, we characterized miRNAs in hops and identified differentially expressed miRNAs in the roots of susceptible and resistant hop cultivars $24 \mathrm{~h}$ after inoculation with the phytopathogenic fungus $V$. nonalfalfae. We identified 56 known miRNAs belonging to 30 different miRNA families and 43 novel miRNAs. In response to Verticillium inoculation, we identified seven and six differentially expressed miRNAs in the susceptible and resistant hop cultivars, respectively, and 11 differentially expressed miRNAs when comparing the susceptible and resistant hop cultivars. The hop cultivars respond to inoculation by altering the expression of different miRNAs. In silico target analysis revealed a total of 49 transcripts that are regulated by differentially expressed miRNAs. According to the gene ontology enrichment analysis, the targets in the susceptible cultivar are involved in protein retention in ER lumen, vesicle-mediated transport and pigment biosynthetic process, etc. In the resistant cultivar, the targets are involved in the auxin-activated (hormonal) signalling pathway and regulation of DNA-templated transcription. The obtained results suggest that miRNAs may play an important role in response and resistance to Verticillium in the resistant hop cultivar. The underlying mechanism related to the observed resistance of the cultivar Wye Target is likely related to miRNA regulation, through which the cultivar modulates biological processes that initiate the growth, development and de novo formation of roots and their vascular tissues when inoculated with $V$. nonalfalfae, which was not observed in the susceptible hop cultivar. The latter shows differential expression of miRNAs, which regulate genes involved in transcriptional reprogramming, leading to changes in metabolism and resulting in unsuccessful defence and the death of the plant. 
Supplementary Materials: The following are available on-line at https:/ / www.mdpi.com/article/ 10.3390/plants10091883/s1, Table S1: Known miRNAs identified in Humulus lupulus. miRNAs were predicted in hop draft genome sequences obtained from HopBase using the miRNA prediction pipeline (miR-PREFeR), followed by alignment of predicted mature miRNA sequences with known miRNAs deposited in miRBase; Table S2: Novel miRNAs identified in Humulus lupulus. miRNAs were predicted in hop draft genome sequences obtained from HopBase using the miRNA prediction pipeline (miR-PREFeR); Table S3: Results of differential expression analysis of miRNAs in roots of the susceptible cultivar Celeia performed by DESeq2; Table S4: Results of differential expression analysis of miRNAs in roots of the resistant cultivar Wye Target performed by DESeq2; Table S5: Results of differential expression analysis of miRNAs between root samples of the susceptible cultivar Celeia and the resistant cultivar Wye Target in response to inoculation with Verticillium nonalfalfae; Table S6: Enriched GO terms of genes targeted by identified differentially miRNAs in the susceptible hop cultivar Celeia. GO analysis was performed with topGO; Table S7: Enriched GO terms of genes targeted by identified differentially miRNAs in the resistant hop cultivar Wye Target. GO analysis was performed with topGO; Table S8: Differentially expressed miRNAs in response to inoculation with $V$. nonalfalfae and their target transcripts in hops. The cleavage sites within the hop transcripts were predicted on-line using psRNATarget analysis server (2017 Update).

Author Contributions: Conceptualization, N.Š. and U.K.; methodology, U.K., S.R., J.J. and N.Š.; validation, U.K., J.J., S.R. and N.Š.; formal analysis, U.K. and N.Š.; investigation, U.K.; resources, S.R., J.J. and N.Š.; writing—original draft, U.K.; writing—review and editing, U.K., J.J., S.R. and N.Š.; visualization, U.K.; supervision, N.Š.; project administration, N.Š.; funding acquisition, U.K. and J.J. All authors have read and agreed to the published version of the manuscript.

Funding: This research was funded by Slovenian Research Agency (SRA-ARRS), grant number 34110 awarded to UK, and SRA-ARRS programme P4-0077 Genetics and Modern Technologies of Crops awarded to the research group at Chair of Genetics, Biotechnology, Statistics and Plant breeding at the University of Ljubljana, Biotechnical Faculty.

Institutional Review Board Statement: Not applicable.

Informed Consent Statement: Not applicable.

Data Availability Statement: The datasets generated and analysed during the current study are available in the NCBI Sequence Read Archive (SRA) repository (https: / www.ncbi.nlm.nih.gov/sra/; accessed on 30 August 2021) under the BioProject accession PRJNA665133 (https://www.ncbi. nlm.nih.gov/bioproject/PRJNA665133; accessed on 30 August 2021) and SRA accession numbers; SRR12696058, SRR12696057, SRR12696054, SRR12696053, SRR12696052, SRR12696051, SRR12696050, SRR12696049, SRR12696048, SRR12696047, SRR12696056, SRR12696055. The hop draft genome was obtained from the HopBase genomic resource which is available at http:/ / hopbase.org (accessed on 30 August 2021) and http:/ / hopbase.cgrb.oregonstate.edu (accessed on 30 August 2021) [30,31] The computationally annotated hop transcriptome is available from our laboratory and the raw NGS sequences are publicly available at NCBI's SRA archive under BioProject number PRJNA342762, BioSample SAMN05767836, SRA run SRR4242068: https: / /www.ncbi.nlm.nih.gov/sra/?term=SRR4 242068 (accessed on 30 August 2021) [82].

Conflicts of Interest: The authors declare no conflict of interest. The funders had no role in the design of the study; in the collection, analyses, or interpretation of data; in the writing of the manuscript, or in the decision to publish the results.

\section{References}

1. Steenackers, B.; De Cooman, L.; De Vos, D. Chemical transformations of characteristic hop secondary metabolites in relation to beer properties and the brewing process: A review. Food Chem. 2015, 172, 742-756. [CrossRef]

2. Hrncic, M.K.; Spaninger, E.; Kosir, I.J.; Knez, Z.; Bren, U. Hop Compounds: Extraction Techniques, Chemical Analyses, Antioxidative, Antimicrobial, and Anticarcinogenic Effects. Nutrients 2019, 11, 257. [CrossRef]

3. Liu, Y.; Gao, X.; Deeb, D.; Arbab, A.S.; Dulchavsky, S.A.; Gautam, S.C. Anticancer agent xanthohumol inhibits IL-2 induced signaling pathways involved in T cell proliferation. J. Exp. Ther. Oncol. 2012, 10, 1-8.

4. Radišek, S. Verticillium Wilt. In Compendium of Hop Diseases and Pests; Mahaffee, W., Pethybridge, S., Gent, D.H., Eds.; The American Phytopathological Society: St. Paul, MI, USA, 2009; pp. 33-36.

5. Radisek, S.; Jakse, J.; Javornik, B. Genetic variability and virulence among Verticillium albo-atrum isolates from hop. Eur. J. Plant Pathol. 2006, 116, 301-314. [CrossRef] 
6. Chisholm, S.T.; Coaker, G.; Day, B.; Staskawicz, B.J. Host-microbe interactions: Shaping the evolution of the plant immune response. Cell 2006, 124, 803-814. [CrossRef]

7. Jones, J.D.G.; Dangl, J.L. The plant immune system. Nature 2006, 444, 323-329. [CrossRef] [PubMed]

8. Fei, Q.L.; Zhang, Y.; Xia, R.; Meyers, B.C. Small RNAs Add Zing to the Zig-Zag-Zig Model of Plant Defenses. Mol. Plant Microbe Interact. 2016, 29, 165-169. [CrossRef] [PubMed]

9. Li, F.; Pignatta, D.; Bendix, C.; Brunkard, J.O.; Cohn, M.M.; Tung, J.; Sun, H.Y.; Kumar, P.; Baker, B. MicroRNA regulation of plant innate immune receptors. Proc. Natl. Acad. Sci. USA 2012, 109, 1790-1795. [CrossRef]

10. D'Ario, M.; Griffiths-Jones, S.; Kim, M. Small RNAs: Big Impact on Plant Development. Trends Plant Sci. 2017, 22, 1056-1068. [CrossRef] [PubMed]

11. Soto-Suarez, M.; Baldrich, P.; Weigel, D.; Rubio-Somoza, I.; San Segundo, B. The Arabidopsis miR396 mediates pathogen-associated molecular pattern-triggered immune responses against fungal pathogens. Sci. Rep. 2017, 7, 1-14. [CrossRef]

12. Navarro, L.; Dunoyer, P.; Jay, F.; Arnold, B.; Dharmasiri, N.; Estelle, M.; Voinnet, O.; Jones, J.D.G. A plant miRNA contributes to antibacterial resistance by repressing auxin signaling. Science 2006, 312, 436-439. [CrossRef] [PubMed]

13. Wong, J.; Gao, L.; Yang, Y.; Zhai, J.X.; Arikit, S.; Yu, Y.; Duan, S.Y.; Chan, V.; Xiong, Q.; Yan, J.; et al. Roles of small RNAs in soybean defense against Phytophthora sojae infection. Plant J. 2014, 79, 928-940. [CrossRef] [PubMed]

14. Natarajan, B.; Kalsi, H.S.; Godbole, P.; Malankar, N.; Thiagarayaselvam, A.; Siddappa, S.; Thulasiram, H.V.; Chakrabarti, S.K.; Banerjee, A.K. MiRNA160 is associated with local defense and systemic acquired resistance against Phytophthora infestans infection in potato. J. Exp. Bot. 2018, 69, 2023-2036. [CrossRef] [PubMed]

15. Jagadeeswaran, G.; Zheng, Y.; Li, Y.F.; Shukla, L.I.; Matts, J.; Hoyt, P.; Macmil, S.L.; Wiley, G.B.; Roe, B.A.; Zhang, W.X.; et al. Cloning and characterization of small RNAs from Medicago truncatula reveals four novel legume-specific microRNA families. New Phytol. 2009, 184, 85-98. [CrossRef]

16. Yin, Z.J.; Li, Y.; Han, X.L.; Shen, F.F. Genome-Wide Profiling of miRNAs and Other Small Non-Coding RNAs in the Verticillium dahliae-Inoculated Cotton Roots. PLoS ONE 2012, 7, e35765. [CrossRef]

17. Cui, X.X.; Yan, Q.; Gan, S.P.; Xue, D.; Dou, D.L.; Guo, N.; Xing, H. Overexpression of gma-miR1510a/b suppresses the expression of a NB-LRR domain gene and reduces resistance to Phytophthora sojae. Gene 2017, 621, 32-39. [CrossRef]

18. Lu, S.F.; Sun, Y.H.; Chiang, V.L. Stress-responsive microRNAs in Populus. Plant J. 2008, 55, 131-151. [CrossRef]

19. Chen, L.; Ren, Y.Y.; Zhang, Y.Y.; Xu, J.C.; Zhang, Z.Y.; Wang, Y.W. Genome-wide profiling of novel and conserved Populus microRNAs involved in pathogen stress response by deep sequencing. Planta 2012, 235, 873-883. [CrossRef]

20. Jakse, J.; Cerenak, A.; Radisek, S.; Satovic, Z.; Luthar, Z.; Javornik, B. Identification of quantitative trait loci for resistance to Verticillium wilt and yield parameters in hop (Humulus lupulus L.). Theor. Appl. Genet. 2013, 126, 1431-1443. [CrossRef]

21. Majer, A.; Javornik, B.; Cerenak, A.; Jakse, J. Development of novel EST-derived resistance gene markers in hop (Humulus lupulus L.). Mol. Breed. 2014, 33, 61-74. [CrossRef]

22. Diwan, N.; Fluhr, R.; Eshed, Y.; Zamir, D.; Tanksley, S.D. Mapping of Ve in tomato: A gene conferring resistance to the broad-spectrum pathogen, Verticillium dahliae race 1. Theor. Appl. Genet. 1999, 98, 315-319. [CrossRef]

23. Kawchuk, L.M.; Hachey, J.; Lynch, D.R.; Kulcsar, F.; van Rooijen, G.; Waterer, D.R.; Robertson, A.; Kokko, E.; Byers, R.; Howard, R.J.; et al. Tomato Ve disease resistance genes encode cell surface-like receptors. Proc. Natl. Acad. Sci. USA 2001, 98, 6511-6515. [CrossRef] [PubMed]

24. Fradin, E.F.; Abd-El-Haliem, A.; Masini, L.; van den Berg, G.C.M.; Joosten, M.H.A.J.; Thomma, B.P.H.J. Interfamily Transfer of Tomato Ve1 Mediates Verticillium Resistance in Arabidopsis. Plant Physiol. 2011, 156, 2255-2265. [CrossRef]

25. Zhang, Z.; Fradin, E.; de Jonge, R.; van Esse, H.P.; Smit, P.; Liu, C.M.; Thomma, B.P.H.J. Optimized Agroinfiltration and VirusInduced Gene Silencing to Study Ve1-Mediated Verticillium Resistance in Tobacco. Mol. Plant Microbe Interact. 2013, 26, 182-190. [CrossRef] [PubMed]

26. Song, Y.; Zhang, Z.; Seidl, M.F.; Majer, A.; Jakse, J.; Javornik, B.; Thomma, B.P.H.J. Broad taxonomic characterization of Verticillium wilt resistance genes reveals an ancient origin of the tomato Ve1 immune receptor. Mol. Plant Pathol. 2017, 18, 195-209. [CrossRef] [PubMed]

27. Mandelc, S.; Timperman, I.; Radisek, S.; Devreese, B.; Samyn, B.; Javornik, B. Comparative proteomic profiling in compatible and incompatible interactions between hop roots and Verticillium albo-atrum. Plant Physiol.Biochem. 2013, 68, 23-31. [CrossRef]

28. Progar, V.; Jakse, J.; Stajner, N.; Radisek, S.; Javornik, B.; Berne, S. Comparative transcriptional analysis of hop responses to infection with Verticillium nonalfalfae. Plant Cell Rep. 2017, 36, 1599-1613. [CrossRef]

29. Cregeen, S.; Radisek, S.; Mandelc, S.; Turk, B.; Stajner, N.; Jakse, J.; Javornik, B. Different Gene Expressions of Resistant and Susceptible Hop Cultivars in Response to Infection with a Highly Aggressive Strain of Verticillium albo-atrum. Plant Mol. Biol. Rep. 2015, 33, 689-704. [CrossRef]

30. Hill, S.T.; Sudarsanam, R.; Henning, J.; Hendrix, D. HopBase: A unified resource for Humulus genomics. Database-Oxf. 2017, 2017. [CrossRef]

31. Padgitt-Cobb, L.K.; Kingan, S.B.; Wells, J.; Elser, J.; Kronmiller, B.; Moore, D.; Concepcion, G.; Peluso, P.; Rank, D.; Jaiswal, P.; et al. A phased, diploid assembly of the Cascade hop (Humulus lupulus) genome reveals patterns of selection and haplotype variation. bioRxiv 2019, 786145. [CrossRef]

32. Lei, J.K.; Sun, Y. miR-PREFeR: An accurate, fast and easy-to-use plant miRNA prediction tool using small RNA-Seq data. Bioinformatics 2014, 30, 2837-2839. [CrossRef] 
33. Kozomara, A.; Griffiths-Jones, S. miRBase: Integrating microRNA annotation and deep-sequencing data. Nucleic Acids Res. 2011, 39, D152-D157. [CrossRef] [PubMed]

34. Sweeney, B.A.; Petrov, A.I.; Burkov, B.; Finn, R.D.; Bateman, A.; Szymanski, M.; Karlowski, W.M.; Gorodkin, J.; Seemann, S.E.; Cannone, J.J.; et al. RNAcentral: A hub of information for non-coding RNA sequences. Nucleic Acids Res. 2019, 47, D221-D229. [CrossRef]

35. Ambros, V.; Bartel, B.; Bartel, D.P.; Burge, C.B.; Carrington, J.C.; Chen, X.M.; Dreyfuss, G.; Eddy, S.R.; Griffiths-Jones, S.; Marshall, M.; et al. A uniform system for microRNA annotation. RNA 2003, 9, 277-279. [CrossRef]

36. Li, W.; Godzik, A. Cd-hit: A fast program for clustering and comparing large sets of protein or nucleotide sequences. Bioinformatics 2006, 22, 1658-1659. [CrossRef] [PubMed]

37. Dai, X.B.; Zhuang, Z.H.; Zhao, P.X.C. psRNATarget: A plant small RNA target analysis server (2017 release). Nucleic Acids Res. 2018, 46, W49-W54. [CrossRef]

38. Yang, K.; Qi, L.; Zhang, Z. Isolation and characterization of a novel wall-associated kinase gene TaWAK5 in wheat (Triticum aestivum). Crop. J. 2014, 2, 255-266. [CrossRef]

39. Li, C.L.; Li, D.Q.; Li, J.; Shao, F.J.; Lu, S.F. Characterization of the polyphenol oxidase gene family reveals a novel microRNA involved in posttranscriptional regulation of PPOs in Salvia miltiorrhiza. Sci. Rep. 2017, 7, 1-15. [CrossRef] [PubMed]

40. Zhao, J.P.; Jiang, X.L.; Zhang, B.Y.; Su, X.H. Involvement of microRNA-Mediated Gene Expression Regulation in the Pathological Development of Stem Canker Disease in Populus trichocarpa. PLoS ONE 2012, 7, e44968. [CrossRef]

41. Gupta, O.P.; Permar, V.; Koundal, V.; Singh, U.D.; Praveen, S. MicroRNA regulated defense responses in Triticum aestivum L. during Puccinia graminis f.sp. tritici infection. Mol. Biol. Rep. 2012, 39, 817-824. [CrossRef]

42. Millar, A.A.; Lohe, A.; Wong, G. Biology and Function of miR159 in Plants. Plants-Basel 2019, 8, 255. [CrossRef] [PubMed]

43. Xue, T.; Liu, Z.H.; Dai, X.H.; Xiang, F.N. Primary root growth in Arabidopsis thaliana is inhibited by the miR159 mediated repression of MYB33, MYB65 and MYB101. Plant Sci. 2017, 262, 182-189. [CrossRef] [PubMed]

44. Palatnik, J.F.; Wollmann, H.; Schommer, C.; Schwab, R.; Boisbouvier, J.; Rodriguez, R.; Warthmann, N.; Allen, E.; Dezulian, T.; Huson, D.; et al. Sequence and expression differences underlie functional specialization of Arabidopsis MicroRNAs miR159 and miR319. Dev. Cell 2007, 13, 115-125. [CrossRef] [PubMed]

45. Allen, R.S.; Li, J.Y.; Stahle, M.I.; Dubroue, A.; Gubler, F.; Millar, A.A. Genetic analysis reveals functional redundancy and the major target genes of the Arabidopsis miR159 family. Proc. Natl. Acad. Sci. USA 2007, 104, 16371-16376. [CrossRef]

46. Choi, M.S.; Woo, M.O.; Koh, E.B.; Lee, J.; Ham, T.H.; Seo, H.S.; Koh, H.J. Teosinte Branched 1 modulates tillering in rice plants. Plant Cell Rep. 2012, 31, 57-65. [CrossRef]

47. Gaudin, A.C.M.; McClymont, S.A.; Soliman, S.S.M.; Raizada, M.N. The effect of altered dosage of a mutant allele of Teosinte branched 1 (tb1-ref) on the root system of modern maize. Bmc Genet. 2014, 15, 23. [CrossRef]

48. Mishra, A.K.; Duraisamy, G.S.; Matousek, J.; Radisek, S.; Javornik, B.; Jakse, J. Identification and characterization of microRNAs in Humulus lupulus using high-throughput sequencing and their response to Citrus bark cracking viroid (CBCVd) infection. BMC Genom. 2016, 17, 919. [CrossRef]

49. Bhogale, S.; Mahajan, A.S.; Natarajan, B.; Rajabhoj, M.; Thulasiram, H.V.; Banerjee, A.K. MicroRNA156: A Potential GraftTransmissible MicroRNA That Modulates Plant Architecture and Tuberization in Solanum tuberosum ssp. andigena. Plant Physiol. 2014, 164, 1011-1027. [CrossRef]

50. Chen, X.; Zhang, Z.; Liu, D.; Zhang, K.; Li, A.; Mao, L. SQUAMOSA promoter-binding protein-like transcription factors: Star players for plant growth and development. J. Integr. Plant Biol. 2010, 52, 946-951. [CrossRef]

51. Stone, J.M.; Liang, X.; Nekl, E.R.; Stiers, J.J. Arabidopsis AtSPL14, a plant-specific SBP-domain transcription factor, participates in plant development and sensitivity to fumonisin B1. Plant J. 2005, 41, 744-754. [CrossRef]

52. Lu, Q.; Shao, F.J.; Macmillan, C.; Wilson, I.W.; van der Merwe, K.; Hussey, S.G.; Myburg, A.A.; Dong, X.M.; Qiu, D.Y. Genomewide analysis of the lateral organ boundaries domain gene family in Eucalyptus grandis reveals members that differentially impact secondary growth. Plant Biotechnol. J. 2018, 16, 124-136. [CrossRef] [PubMed]

53. Ohashi-Ito, K.; Iwamoto, K.; Fukuda, H. LOB DOMAIN-CONTAINING PROTEIN 15 Positively Regulates Expression of VND7, a Master Regulator of Tracheary Elements. Plant Cell Physiol. 2018, 59, 989-996. [CrossRef] [PubMed]

54. Soyano, T.; Thitamadee, S.; Machida, Y.; Chua, N.H. ASYMMETRIC LEAVES2-LIKE19/LATERAL ORGAN BOUNDARIES DOMAIN30 and ASL20/LBD18 Regulate Tracheary Element Differentiation in Arabidopsis. Plant Cell 2008, 20, 3359-3373. [CrossRef] [PubMed]

55. Reusche, M.; Thole, K.; Janz, D.; Truskina, J.; Rindfleisch, S.; Drubert, C.; Polle, A.; Lipka, V.; Teichmann, T. Verticillium Infection Triggers VASCULAR-RELATED NAC DOMAIN7-Dependent de Novo Xylem Formation and Enhances Drought Tolerance in Arabidopsis. Plant Cell 2012, 24, 3823-3837. [CrossRef]

56. Hu, G.; Lei, Y.; Wang, L.; Liu, J.; Tang, Y.; Zhang, Z.; Chen, A.; Peng, Q.; Yang, Z.; Wu, J. The ghr-miR164 and GhNAC100 module participates in cotton plant defence against Verticillium dahliae. bioRxiv 2018, 440826. [CrossRef]

57. Wang, Z.; Xia, Y.; Lin, S.; Wang, Y.; Guo, B.; Song, X.; Ding, S.; Zheng, L.; Feng, R.; Chen, S.; et al. Osa-miR164a targets OsNAC60 and negatively regulates rice immunity against the blast fungus Magnaporthe oryzae. Plant J. 2018, 95, 584-597. [CrossRef]

58. Guo, H.S.; Xie, Q.; Fei, J.F.; Chua, N.H. MicroRNA directs mRNA cleavage of the transcription factor NAC1 to downregulate auxin signals for Arabidopsis lateral root development. Plant Cell 2005, 17, 1376-1386. [CrossRef]

59. Bari, R.; Jones, J. Role of plant hormones in plant defence responses. Plant Mol. Biol. 2009, 69, 473-488. [CrossRef] 
60. Tiwari, S.B.; Hagen, G.; Guilfoyle, T. The roles of auxin response factor domains in auxin-responsive transcription. Plant Cell 2003, 15, 533-543. [CrossRef]

61. Wang, J.W.; Wang, L.J.; Mao, Y.B.; Cai, W.J.; Xue, H.W.; Chen, X.Y. Control of root cap formation by microRNA-targeted auxin response factors in Arabidopsis. Plant Cell 2005, 17, 2204-2216. [CrossRef]

62. Mallory, A.C.; Bartel, D.P.; Bartel, B. MicroRNA-directed regulation of Arabidopsis AUXIN RESPONSE FACTOR17 is essential for proper development and modulates expression of early auxin response genes. Plant Cell 2005, 17, 1360-1375. [CrossRef] [PubMed]

63. Li, Y.; Zhao, S.L.; Li, J.L.; Hu, X.H.; Wang, H.; Cao, X.L.; Xu, Y.J.; Zhao, Z.X.; Xiao, Z.Y.; Yang, N.; et al. Osa-miR169 Negatively Regulates Rice Immunity against the Blast Fungus Magnaporthe oryzae. Front. Plant Sci. 2017, 8, 2. [CrossRef] [PubMed]

64. Sorin, C.; Declerck, M.; Christ, A.; Blein, T.; Ma, L.; Lelandais-Briere, C.; Njo, M.F.; Beeckman, T.; Crespi, M.; Hartmann, C. A miR169 isoform regulates specific NF-YA targets and root architecture in Arabidopsis. New Phytol. 2014, 202, 1197-1211. [CrossRef] [PubMed]

65. Dodds, P.N.; Rathjen, J.P. Plant immunity: Towards an integrated view of plant-pathogen interactions. Nat. Rev. Genet. 2010, 11, 539-548. [CrossRef] [PubMed]

66. Yin, C.T.; Ramachandran, S.R.; Zhai, Y.; Bu, C.Y.; Pappu, H.R.; Hulbert, S.H. A novel fungal effector from Puccinia graminis suppressing RNA silencing and plant defense responses. New Phytol. 2019, 222, 1561-1572. [CrossRef] [PubMed]

67. Pulsifer, I.P.; Kluge, S.; Rowland, O. Arabidopsis LONG-CHAIN ACYL-COA SYNTHETASE 1 (LACS1), LACS2, and LACS3 facilitate fatty acid uptake in yeast. Plant Physiol. Biochem. 2012, 51, 31-39. [CrossRef]

68. Deng, Y.X.; Lu, S.F. Biosynthesis and Regulation of Phenylpropanoids in Plants. CRC Crit. Rev. Plant Sci. 2017, 36, 257-290. [CrossRef]

69. Bonar, N.; Liney, M.; Zhang, R.X.; Austin, C.; Dessoly, J.; Davidson, D.; Stephens, J.; McDougall, G.; Taylor, M.; Bryan, G.J.; et al. Potato miR828 Is Associated With Purple Tuber Skin and Flesh Color. Front. Plant Sci. 2018, 9, 1742. [CrossRef]

70. Aasland, R.; Stewart, A.F.; Gibson, T. The SANT domain: A putative DNA-binding domain in the SWI-SNF and ADA complexes, the transcriptional co-repressor N-CoR and TFIIIB. Trends Biochem. Sci. 1996, 21, 87-88. [CrossRef]

71. Chi, M.; Liu, C.M.; Su, Y.Q.; Tong, Y.W.; Liu, H.Y. Bioinformatic prediction of upstream microRNAs of PPO and novel microRNAs in potato. Can. J. Plant Sci. 2015, 95, 871-877. [CrossRef]

72. Ren, G.H.; Wang, B.J.; Zhu, X.D.; Mu, Q.; Wang, C.; Tao, R.; Fang, J.G. Cloning, expression, and characterization of miR058 and its target PPO during the development of grapevine berry stone. Gene 2014, 548, 166-173. [CrossRef]

73. Schindler, C.; Chen, Y.; Pu, J.; Guo, X.L.; Bonifacino, J.S. EARP is a multisubunit tethering complex involved in endocytic recycling Nat. Cell Biol. 2015, 17, 639-650. [CrossRef] [PubMed]

74. Flajsman, M.; Radisek, S.; Javornik, B. Pathogenicity Assay of Verticillium nonalfalfae on Hop Plants. Bio-Protoc. 2017,7 , e2171. [CrossRef]

75. Langmead, B.; Trapnell, C.; Pop, M.; Salzberg, S.L. Ultrafast and memory-efficient alignment of short DNA sequences to the human genome. Genome Biol. 2009, 10, R25. [CrossRef]

76. Axtell, M.J.; Meyers, B.C. Revisiting Criteria for Plant MicroRNA Annotation in the Era of Big Data. Plant Cell 2018, 30, $272-284$. [CrossRef] [PubMed]

77. Langmead, B.; Salzberg, S.L. Fast gapped-read alignment with Bowtie 2. Nat. Methods 2012, 9, 357-359. [CrossRef] [PubMed]

78. Lorenz, R.; Bernhart, S.H.; Siederdissen, C.H.Z.; Tafer, H.; Flamm, C.; Stadler, P.F.; Hofacker, I.L. ViennaRNA Package 2.0. Algorithms Mol. Biol. 2011, 6, 26. [CrossRef] [PubMed]

79. Zhang, B.H.; Pan, X.P.; Cox, S.B.; Cobb, G.P.; Anderson, T.A. Evidence that miRNAs are different from other RNAs. Cell Mol. Life Sci. 2006, 63, 246-254. [CrossRef] [PubMed]

80. R Core Team. R: A Language and Environment for Statistical Computing, 3.5.1; R Foundation for Statistical Computing: Vienna, Austria, 2017.

81. Love, M.I.; Huber, W.; Anders, S. Moderated estimation of fold change and dispersion for RNA-seq data with DESeq2. Genome Biol. 2014, 15, 1-21. [CrossRef]

82. Pokorn, T.; Radisek, S.; Javornik, B.; Stajner, N.; Jakse, J. Development of hop transcriptome to support research into host-viroid interactions. PLoS ONE 2017, 12, e0184528. [CrossRef]

83. Alexa, A.; Rahnenfuhrer, J. topGO: Enrichment Analysis for Gene Ontology, 2.40.0; Bioconductor: Buffalo, NY, USA, 2020.

84. Shannon, P.; Markiel, A.; Ozier, O.; Baliga, N.S.; Wang, J.T.; Ramage, D.; Amin, N.; Schwikowski, B.; Ideker, T. Cytoscape: A software environment for integrated models of biomolecular interaction networks. Genome Res. 2003, 13, 2498-2504. [CrossRef] [PubMed]

85. Doncheva, N.T.; Morris, J.H.; Gorodkin, J.; Jensen, L.J. Cytoscape StringApp: Network Analysis and Visualization of Proteomics Data. J. Proteome Res. 2019, 18, 623-632. [CrossRef] [PubMed] 